\title{
In Vitro Measurement of Enzymatic Markers as a Tool to Detect Mouse Cardiomyocytes Injury
}

\author{
Elen Mello de Souza, Andrea Henriques-Pons, Christian Bailly*, Amelie Lansiaux*, \\ Tânia Cremonine Araújo-Jorge, Maria de Nazaré Correia Soeiro/ ${ }^{+}$
}

\begin{abstract}
Laboratório de Biologia Celular, Departamento de Ultra-estrutura e Biologia Celular, Instituto Oswaldo Cruz-Fiocruz, Av. Brasil 4365, 21040-900 Rio de Janeiro, RJ, Brasil *INSERM U-524/Laboratoire de Pharmacologie Antitumorale, Centre Oscar Lambret, Institute de Recherches sur le Cancer de Lille, Lille, France
\end{abstract}

Primary cultures of cardiomyocytes represent a useful model for analyzing cardiac cell biology as well as pathogenesis of several cardiovascular disorders. Our aim was to standardize protocols for determining the damage of cardiac cells cultured in vitro by measuring the creatine kinase and its cardiac isotype and lactate dehydrogenase activities in the supernatants of mice cardiomyocytes submitted to different protocols of cell lysis. Our data showed that due to its higher specificity, the cardiac isotype creatine kinase was the most sensitive as compared to the others studied enzymatic markers, and can be used to monitor and evaluate cardiac damage in in vitro assays.

Key words: creatine kinase - in vitro assays - cardiac damage

The cellular damage of heart cells plays an important role in the pathogenesis of several cardiovascular disorders. It is accepted that the non-invasive identification and localization of myocardial lesions helps in the diagnosis of these disorders, as well as in prognosis and assessment of treatment response (Flotats \& Carrio 2003). Initial attempts to directly visualize the area of myocardial injury were based on the recognition of myocardial infarction with hot spot imaging agents (Botvinic 1990). Biochemical methods represent an important tool for the diagnostic of cardiac injury. Among the commercially available markers, the most used non-enzymatic markers are the myoglobin, the troponin $\mathrm{T}$, and troponin I molecules. While myoglobin is a non-specific indicator of myocardial damage, its diagnostic value relies on its early appearance in the blood (Bhayana \& Henderson 1995). The high sensitivity and specificity of the cardiac troponins make them one of the most used biochemical non-enzymatic markers for diagnosing the myocardial damage (Amit et al. 2003). However, troponin measurements appear to have an equivalent diagnostic accuracy compared to the creatine kinase cardiac isoenzyme (CK-MB) marker for diagnosing myocardial necrosis such as reported in patients with cocaine-associated chest pain and suspected infarction (Kontos et al. 2002). Creatine kinase (CK; EC 2.7.3.2) is a meaningful enzyme regulator of high-energy phosphate production and utilization in contractile tissues (Takagi et al. 2001). CK can be found in brain, in

Financial support: Fundação Carlos Chagas Filho de Amparo à Pesquisa do Estado do Rio de Janeiro, Conselho Nacional Desenvolvimento Científico e Tecnológico, Papes III-Fiocruz, Convention INSERM-Fiocruz

${ }^{+}$Corresponding author. Fax: +55-21-22604434. E-mail: soeiro@ioc.fiocruz.br

Received 29 March 2004

Accepted 20 September 2004 striated and smooth muscles, and their serum levels have been largely used as indicator of tissue injury especially in the skeletal and cardiac tissues. CK has three cytoplasmatic isozymes (CK-MM, CK-MB, and CK-BB) and due to its specific localization in the tissues (skeletal muscle, cardiac muscle, and brain, respectively), CK measurements provide substantial information concerning cellular injury in acute skeletal muscle diseases, myocardial infarction, brain damage, and malignant tumor of the gastrointestinal tract (Takagi et al. 2001). CK-MB activity can be measured either by enzyme activity or mass concentration. Other markers with enzymatic activity that can be assayed include the lactate dehydrogenase (LDH) (Zhang et al. 2000) and its isoforms, and the aspartate aminotransferase (Nishimura \& Yazaki 1995), which offer the lower costs. However, both are supplanted by the CK detection (together isoenzymes and isoforms) due to aforementioned superior tissue specificity. The biochemical detection of CK-MB plasma levels has been used to monitor the occurrence of experimental chagasic myocarditis an the kinetic of the necrotic heart lesions found in the histopathological sections were clearly related to the increase of CK-MB in the plasma (de Souza et al. 2000).

Since feasible enzymatic biochemical markers are pivotal for detecting cellular injury in cardiac cells grown in culture, our present aim was to standardize protocols for determining creatine kinase, its cardiac isoenzyme, and lactacte dehydrogenase activities in the supernatants of primary cultures of cardiomyocytes submitted to different protocols of cell lysis.

\section{MATERIALS AND METHODS}

Cell cultures - Primary cultures of mice embryonic cardiomyocytes (CM) were obtained as previously described (Meirelles et al. 1986). Briefly, heart from 18-dayold Swiss mice embryos were isolated and the ventricular muscle cut into small pieces before submitting to a serial of enzymatic dissociation steps employing $0.01 \%$ collagenase $0.025 \%$ trypsin diluted in phosphate-buffered sa- 
line, $\mathrm{pH} 7.0$ (PBS). Enzyme inactivation was achieved by adding $20 \%$ fetal calf serum and the released cells were centrifuged for $7 \mathrm{~min}$ at $650 \mathrm{~g}$. The resulted pellet was ressuspended in Dulbecco's modified medium (DMEM) supplemented with $10 \%$ horse serum, $5 \%$ fetal bovine serum, $2.5 \mathrm{mM} \mathrm{CaCl}_{2}, 1 \mathrm{mM} \mathrm{L}$-glutamine, and $2 \%$ chicken embryo extract. The purified heart cells were then platted for $20 \mathrm{~min}$ in $0.01 \%$ gelatin-coated culture flasks to allow the adhesion of fibroblasts. Next, the cellular density of the cardiomyocyte-enriched supernatant was established and the cells seeded at different cell densities per well into 96-well culture plates previously coated with $0.01 \%$ gelatin. Cultures were maintained at $37^{\circ} \mathrm{C}$ in an atmosphere of $5 \% \mathrm{CO}_{2}$ in DMEM supplemented as above discriminated. Peritoneal mouse resident macrophages were cultivated as described elsewhere (Araújo-Jorge et al. 1989) and were used as negative controls for CK-MB measurements. All assays described here were run 3 to 5 times at least in triplicate. All procedures were carried out in accordance with the guidelines established by the Fiocruz Committee of Ethics for the Use of Animals, resolution 242/99.

Fluorescent analysis - The CM were fixed for $20 \mathrm{~min}$ with $4 \%$ paraformaldehyde, permeabilized with $0.2 \%$ Triton-X 100 in PBS for 2 to 3 min, incubated with phalloidinFITC and DAPI for actin staining and DNA respectively. The samples were then mounted with DABCO to prevent fading and analyzed at Zeiss photomicroscope equipped with epifluorescence.

Ultrastructural analysis - For transmission electron microscopy procedure, the $\mathrm{CM}$ were fixed for $1 \mathrm{~h}$ at $4^{\circ} \mathrm{C}$ with $2.5 \%$ GA in $0.01 \mathrm{M} \mathrm{Na}$ cacodylate buffer, $\mathrm{pH}$ 7.2, rinsed in the same buffer. The CM were then post-fixed with $1 \% \mathrm{O}_{\mathrm{s}} \mathrm{O}_{4}$, dehydrated in acetone and embedded in Epon. Thin sections were stained with uranyl acetate and lead citrate and examined in an EM 10C Zeiss microscope.

Cell lysis - For the initial standardization, $7.5 \times 10^{4}$ uninfected $\mathrm{CM}$ platted for $48 \mathrm{~h}$ at $37^{\circ} \mathrm{C}$, were washed three times with $0.01 \mathrm{M}$ PBS to remove the medium and serum sources. Then the monolayers were treated for $20 \mathrm{~min}$ at room temperature (RT) with agents $(50 \mu \mathrm{l} /$ well) to induce cellular damage: deionized $\mathrm{H}_{2} \mathrm{O}$ and different concentration of Triton X-100 (0.25-2\%) diluted in $0.1 \%$ sodium citrate. As negative control for the cell lysis, the CM was incubated in PBS alone. After the incubation, the culture supernatant from each well was transferred to another 96well-microtiter assay plate and further incubated with the reagent solution kit for detection of CK, CK-MB, and LDH activities. To explore the correlation between the cell density and the release of the CK-MB activity, CM were seeded at different densities $\left(0.3-20 \times 10^{4}\right.$ cells/well $)$, washed and treated with $2 \%$ Triton X-100 (T2) following the same protocols as described above. In order to improve the cellular damage caused by $\mathrm{T} 2$, the plates were submitted or not to centrifugation at $430 \mathrm{~g}$ during the incubation time. Peritoneal macrophages were always employed in parallel assays as negative controls.

Detection of creatine kinase (E.C. 2.7.3.2), creatine kinase cardiac isoenzyme, and lactate dehydrogenase activities - All the enzymatic activities were performed using commercial Kits (Merck, Darmstadt, Germany). For detection of the CK-MB, CK, and $\mathrm{LDH}$, the CM were washed and treated as described above and then $5 \mu \mathrm{l}$ of the culture supernatant were transferred to another 96well-microtiter assay plate and further incubated for 3, 5 or 1 min at RT with 125,125 or $250 \mu 1$ of the reagent solution kit for detection of CK, CK-MB and LDH, respectively. In some approaches for CK-MB detection, we assayed different amounts of the cardiac culture supernatant (5 to $20 \mu \mathrm{l}$ ) keeping the same amount of the reaction solution $(125 \mu \mathrm{l})$. The absorbance was read at each minute using a $340 \mathrm{~nm}$ filter using a micro ELISA automatic reader (Titertek Plus-ICN/Flow) and the 6, 5, or 3 readings were applied for the determination of CK-MB, CK, and LDH activities, respectively. The resulted enzyme activities were expressed in mean $\Delta \mathrm{OD} / \mathrm{min} \times 10-3$ as described elsewhere (de Souza et al. 2000).

Viability of the host cells - To assess the cardiac cell viability, the CM cultures seeded at different cellular densities were submitted or not to $2 \%$ Triton X-100 treatment as described above, washed and further incubated for 1 $\mathrm{min} / \mathrm{RT}$ with $0.3 \mathrm{mg} / \mathrm{ml}$ trypan blue dye diluted in PBS following by the analysis and quantification at the light microscopy (Zeiss photomicroscope).

\section{RESULTS}

After $48 \mathrm{~h}$ of plating, the differentiated cardiac myocytes cultured in vitro show morphological (Fig. 1 A-C) and physiological patterns of in vivo cardiac cells including spontaneous contraction, striated myofibrils, numerous mitochondria profiles, caveolae, gap junctions and intercalated disks. Thus, the present cardiac myocyte primary cultures represent an important tool for cardiac cell biological research as reported (Meirelles et al. 1986, de Souza et al. 2003). Since CK-MB is one of the most used enzymatic marker for the diagnostic of cardiac damage, we initiated our study by standardizing the release of CKMB activity in the supernatant of cardiomyocytes cultures submitted to different protocols of cell lysis: by using water and by increasing concentration of pore forming detergent such as Triton X-100. All the employed protocols lead to the release of CK-MB into the culture supernatant, changing from 0.3 to 63 the mean OD differences observed every minute, confirming the cardiac lysis (Table I). The damage could also be detected by light microscopy using the trypan blue dye exclusion assay that revealed the total loss of the cardiac cell viability (data not shown). Among the protocols, the treatment for 20 min with the detergent $2 \%$ Triton X-100 was the most effective, reaching mean levels of $63 \Delta \mathrm{OD} / \mathrm{min} \times 10-3$. As expected, the incubation of the cells only with PBS did not result in any release of the enzymatic activity (Table I). To assay the sensitivity of the methodology, different densities of CM were seeded and submitted to T2 incubation. We found a reliable and positive correlation between the cell density and CK-MB activity: $\Delta \mathrm{OD} / \mathrm{min}$ values increased according to the crescent number of cardiomyocytes (Table II) showing a correspondence between the injury level and the amount of released molecules. However, since the amount of CK-MB activity was almost undetectable when concentrations of $\mathrm{CM}$ were low as $1.2 \times 10^{4}$ (Table II), we wonder if the centrifugation pro- 
cedure during the incubation with the detergent could enhance the release of the marker to the extracellular medium by improving the cellular damage caused by $\mathrm{T} 2$. The detergent treatment along with the centrifugation procedure released higher amounts of CK-MB as compared to correspondent non-centrifuged CM samples (Table II).
The addition of the centrifugation step enhanced the sensitivity of the method for measuring the CK-MB activity even employing very low densities of CM such as 0.3 $\mathrm{x} 10^{4}$, reaching $\Delta \mathrm{OD} / \mathrm{min} \times 10-3$ mean readings of 57 , which was previously undetectable when only the detergent was used, while the centrifugation step alone in the absence
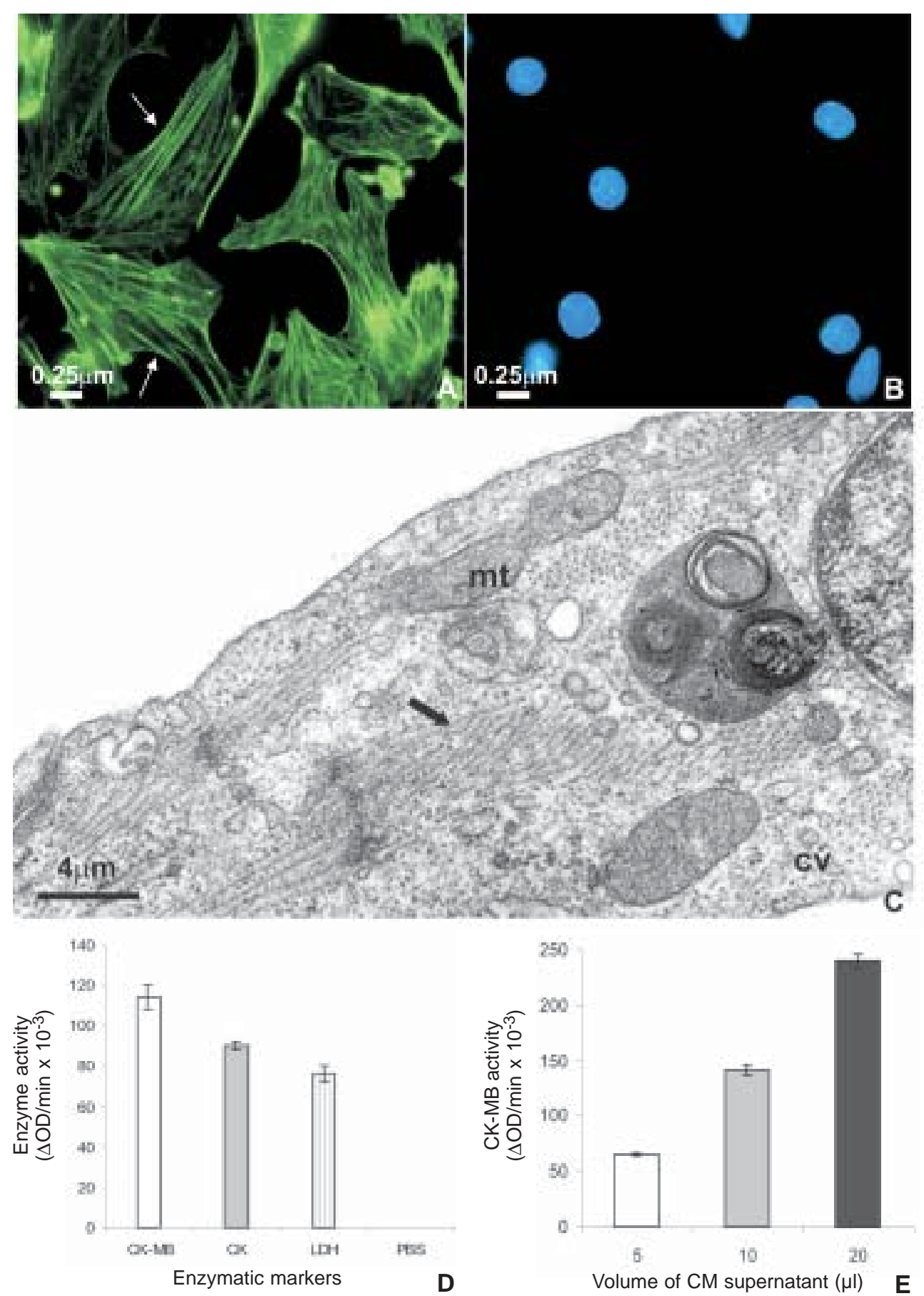

Fig. 1: cell morphology (A-C) of primary cultures of mice cardiac myocytes. A and B: fluorescent micrographs of cardiomyocytes (CM) showing sarcomeres labeled with phalloidin-FITC (A, arrows) and the respective nuclei stained with DAPI (B); C: transmission electron microscopy image of typical cardiac myocytes showing myofibrils $(\rightarrow)$, caveolae (cv), and mitochondria (mit); D and E: evaluation of cellular damage in CM through enzymatic markers; D: CK, CK-MB, and LDH activities in the supernatant of $7.5 \times 10^{4}$ cultured cells upon $2 \%$ Triton-X 100 treatment. Note the higher specificity of CK-MB activity with higher $\Delta \mathrm{OD} / \mathrm{min}$ values. The PBS represents the measurements in the absence of any enzyme substrate; E: measurements of CK-MB activities employing different supernatants volumes of $7.5 \times 10^{4}$ cultured cardiomyocytes submitted to treatment with $2 \%$ Triton-X 100 . 
TABLE I

Effect on the CK-MB release from $7.5 \times 104$ cultured cardiac myocytes after different treatments. The resulted enzyme activities were expressed in mean of $\Delta O D / m i n \times 10^{-3}$

\begin{tabular}{lc}
\hline Agent of cell lysis & $\Delta \mathrm{OD} / \mathrm{min} \times 10^{-3}$ \\
\hline PBS & $0.3 \pm 1$ \\
$\mathrm{H} 2 \mathrm{O}$ & $19 \pm 4$ \\
$0.25 \% \mathrm{~T}$ & $50 \pm 6$ \\
$1 \% \mathrm{~T}$ & $56 \pm 3$ \\
$2 \% \mathrm{~T}$ & $63 \pm 3$ \\
\hline
\end{tabular}

T: Triton $\mathrm{X}-100$

of T2 did not induce detectable CK-MB release (Table II). To assess the specificity of the CK-MB release methodology, we performed similar assays using peritoneal macrophages. As expected due to the CK-MB cardiac localization, CK-MB release was negligible in the supernatant of lysed macrophages as compared to the CM, even when the double of the maximum cellular density were used (Table II). As other enzymatic markers have been used in clinical trials for detecting tissue damage, we next compared the use of different markers for measuring cardiomyocytes injury. Our data show that we successfully detected all the enzymatic activities into the CM supernatant after incubation for $20 \mathrm{~min}$ with $\mathrm{T} 2$, reaching mean values of 114,90 , and $76 \Delta \mathrm{OD} / \mathrm{min} \times 10-3$ for $\mathrm{CK}$ $\mathrm{MB}, \mathrm{CK}$, and LDH, respectively (Fig. $1 \mathrm{D})$. Since the former enzyme are only expressed by cardiac muscle cells, our assays confirmed the higher specificity and sensitivity for CK-MB activity, which reached the higher $\Delta \mathrm{OD} / \mathrm{min}$ levels in CM but was undetectable in the supernatant of lysed macrophage cultures, which have been used as negative controls (Table II). We finally tested the correlation between supernatant volume and amount of the enzyme substrate that was offerred and found that increasing the supernatant volume lead to higher $\Delta \mathrm{OD} / \mathrm{min}$ levels (Fig. 2E).

\section{DISCUSSION}

The choice of a biochemical marker for detecting cellular damage of the cardiomyocytes depends on (i) its specificity towards the myocardial cells, (ii) its fast release after cell injury, (iii) the linear correspondence between the injury level and the amount of released molecules, (iv) its easy measurement by automatic methods, and finally (v) its low cost. The measurements of the CKMB levels in the CM supernatant as described here is a rapid, low cost and easy methodology, presenting reliable correspondence between the cell density and the marker release. This isoenzyme still represents one of the first choices for detecting the cardiac cells injury in the plasma analysis of patients displaying different types of heart diseases (Tietz 1980). We found that this method also presents a high specificity towards cardiac cells cultured in vitro since undetectable levels were consistently found when other cell types were used such as peritoneal macrophages. The parallel analysis of the CM morphological by light microscopy along with the trypan blue exclusion analysis showed a good correlation between cell viability and CK-MB detection. In an ex-vivo study, it has been demonstrated a clear correlation of CK release and impaired cardiomyocytes necrosis but not apoptosis after verapamil treatment of rat hearts submitted to ischemia and reperfusion procedures (Di Napoli et al. 2002). Besides, it has been suggested that CK isoenzymes could be employed as the index for the infection and necrosis of the muscle in electrical injured patients due to its high specificity and sensitivity (Liu \& Xie 2002). Literature data show that in vitro models can provide a rapid, precise and relevant tool to reduce and replace some in vivo studies (Person 1986). Cell injury in cultured cells has been approached by several methodologies including morphological and functional assays (Civiale et al. 2003), the measurement of lactate dehydrogenase leakage, and the reduction of the tetrazolium salt to formazan by mitochondrial metabolic activity (Huhtala et al. 2002). Our study described a sensitive method for detecting cellular damage in the supernatant of primary cultures of cardiac myocytes. Among the markers that we tested, LDH, CK, and its cardiac isoenzyme $\mathrm{CK}-\mathrm{MB}$, the latter proved to be the most specific and assured methodology for detecting cardiac injury in vitro. Cytotoxicity studies employing culture systems have been performed and some employ detergents such as triton X-100 to induce cellular mem-

TABLE II

Effect of centrifugation procedure $(430 \mathrm{~g})$ and correlation between the cell density of cardiac myocyte and the corresponding CK-MB release after $2 \%$ triton treatment for $20 \mathrm{~min}$

\begin{tabular}{lccr}
\hline & \multicolumn{3}{c}{ CK-MB activity $\left(\Delta \mathrm{OD} / \mathrm{min} \times 10^{-3}\right)$} \\
\cline { 2 - 4 } Cell density $\left(10^{4}\right)$ & 0 & Cardiomyocyte & Macrophage \\
\cline { 2 - 4 } & Not centrifuged & Centrifuged & centrifuged \\
\hline 0.3 & $1 \pm 1$ & $57 \pm 3$ & nd \\
0.6 & $2 \pm 0$ & $62 \pm 5$ & 0 \\
1.2 & $8 \pm 3$ & $66 \pm 5$ & 0 \\
2.5 & $19 \pm 3$ & $71 \pm 4$ & $2 \pm 0$ \\
5 & $63 \pm 3$ & $92 \pm 2$ & nd \\
7.2 & $85 \pm 10$ & $114 \pm 6$ & $4 \pm 1$ \\
10 & nd & $170 \pm 2$ & $7 \pm 1$ \\
20 & & nd & 7 \\
\hline
\end{tabular}

nd: not done 
brane disruption and rapid lysis of the cultured cells (Dias et al. 2003). Presently, we describe a simple method for detecting cellular damage in $\mathrm{CM}$ indicated by the release of CK-MB, CK, and LDH in the cell culture supernatant upon the use of the non-ionic surfactant agent Triton $\times 100$. This methodology that identifies the loss of cardiac myocyte viability can be used in several in vitro approaches such as the assessment of irritant and corrosive properties of chemicals, during cytotoxicity assays employing heterotypic interaction of cardiomyocytes with immune effectors cells as well during the analysis of pathological damage caused by pathogens.

\section{REFERENCES}

Amit G, Gilutz H, Zahger D 2003. The use of cardiac troponins in acute coronary syndromes. Harefuah 142: 109-114.

Araújo-Jorge TC, Sampaio ED, de Souza W, Meirelles MNL 1989. T. cruzi: the effect of variations in experimental conditions on the levels of macrophage infection "in vitro". Parasitol Res 75: 257-263.

Bhayana V, Henderson AR 1995. Biochemical markers of myocardial damage. Clin Biochem 28: 1-29.

Botvinick EH 1990. "Hot spot" imaging agents for acute myocardial infarction. J Nucl Med 31: 143-146.

Civiale C, Paladino G, Marino C, Trombetta F, Pulvirenti T, Enea V 2003. Multilayer primary epithelial cell culture from bovine conjunctiva as a model for in vitro toxicity tests. Ophthalmic Res 35: 126-136.

de Souza AP, Olivieri BP, De Castro SL, Araújo-Jorge TC 2000. Enzymatic markers of heart lesion in mice infected with Trypanosoma cruzi and submitted to benznidazole chemotherapy. Parasitol Res 86: 800-808.

de Souza EM, Araújo-Jorge TC, Bailly C, Lansiaux A, Batista MM, Oliveira GM, Soeiro MNC 2003. Host and parasite apoptosis following Trypanosoma cruzi infection in in vitro and in vivo models. Cell Tissue Res 314: 223-235.

Di Napoli P, Taccardi AA, Grill A, Felaco M, Di Gioacchino L, De Caterina R, Barsotti A 2002. Verapamil reduces coro- nary endothelium damage and cardiomyocyte necrosis but not apoptosis after ischemia and reperfusion: ex-vivo study in rat hearts. Int J Immunopathol Pharmacol 15: 225-232.

Dias N, Mortara RA, Lima N 2003. Morphological and physiological changes in Tetrahymena pyriformis for the in vitro cytotoxicity assessment of Triton X-100. Toxicol In Vitro 17: 357-366.

Flotats A, Carrio I 2003. Non-invasive in vivo imaging of myocardial apoptosis and necrosis. Eur J Nucl Med Mol Imaging 30: 615-630.

Huhtala A, Mannerstrom M, Alajuuma P, Nurmi S, Toimela T, Tahti H, Salminen L, Uusitalo H 2002. Comparison of an immortalized human corneal epithelial cell line and rabbit corneal epithelial cell culture in cytotoxicity testing. J Ocul Pharmacol Ther 18: 163-175.

Kontos MC, Anderson FP, Ornato P, Tatum JL, Jesse RL 2002. Utility of troponin I in patients with cocaine-associated chest pain. Acad Emerg Med 9: 1007-1013.

Liu S, Xie W 2002. Clinical significance of the change of serum CK-MM in electrical injured patients. Zhonghua Shao Shang Za Zhi 18: 226-228.

Meirelles MNL, Araújo-Jorge TC, Miranda CF, De Souza W, Barbosa HS 1986. Interaction of T. cruzi with heart muscle cells: ultrastructural and cytochemical analysis of endocytic vacuole formation and effect upon myogenesis in vitro. Eur J Cell Biol 41: 198-206.

Nishimura H, Yazaki Y 1994. Biochemical tests for diagnosis of acute myocardial infarction and estimation of infarct size. Nippon Rinsho 52: 755-759.

Pearson RM 1986. In-vitro techniques: can they replace animal testing? Hum Reprod 1: 559-560.

Takagi Y, Yasuhara T, Gomi K 2001. Creatine kinase and its isozymes. Rinsho Byori 116: 52-61.

Tietz NW 1980. Present and future trends in selected areas of clinical enzymology. J Clin Chem Clin Biochem 18: 763769.

Zhang JG, Ghosh S, Ockleford CD, Galiñanes M 2000. Characterization of an in vitro model for the study of the short and prolonged effects of myocardial ischaemia and reperfusion in man. Clin Sci 99: 443-453. 
